\title{
A clinical study of radiation cataract formation in adult life following $\gamma$ irradiation of the lens in early childhood
}

Gunilla Wilde, Johan Sjöstrand

\begin{abstract}
Aims-To analyse long term effects on the lens of radium irradiation during infancy. Methods-An infant cohort $(n=20$, median age 6 months) treated for skin haemangioma with one or two radium-226 needles located at or within the orbital rim was examined 30 to 45 years after $\gamma$ radiation. Detailed information about the treatment procedure was available for all cases. Subcapsular opacities were graded semiquantitatively according to a scale based on extent and density of the opacities.

Results-A high prevalence of light to moderate posterior, subcapsular, and cortical cataract formation was found in the lenses on the treated side irradiated with a mean dose ranging from approximately 1 to $8 \mathrm{~Gy}$. The cataract formation increased as a function of dose. The presence of subcapsular punctate opacities and vacuoles in the lenses on the untreated side receiving irradiation of an estimated dose varying around $0.1 \mathrm{~Gy}$ indicates $\mathrm{a}$ higher sensitivity than expected.

Conclusion-The growing lens during infancy is sensitive to radium irradiation at doses lower than those previously stated. The eye lens seems suitable for studies of effects of low dose radiation since damaged cells are retained in the lens for a lifetime.
\end{abstract}

(Br F Ophthalmol 1997;81:261-266)

Institute of Clinical

Neuroscience,

Department of

Ophthalmology,

Sahlgrenska University

Hospital, Gothenburg,

Sweden

G Wilde

J Sjöstrand

Correspondence to:

Dr Johan Sjöstrand, Institute of Clinical Neuroscience,

Department of

Ophthalmology, Sahlgrenska

University Hospital, S-413

45 Göteborg, Sweden.

Accepted for publication 25 November 1996 The lens opacification is believed to be initiated by radiation injury to the lens epithelium near the equator, with interference with normal lens fibre differentiation. ${ }^{34}$ The cataractogenic dose of various qualities of radiation and the dose-response relation of the human lens as well as age dependence are not well documented. The estimated minimal or threshold dose required for lenticular opacifi- cation differs in various surveys as a result of variation in observation method used and individual sensitivity. ${ }^{5-8}$ Furthermore, few quantitative studies are available regarding long term effects of irradiation on infants and children with respect to threshold dose for induction of cataract and the length of the latent periodthat is, time interval between treatment and appearance of lens opacities. Clinical studies on human adults have presented uncertain data concerning the length of the latency period, with it ranging from 0.5 to 35 years with shorter latencies as well as a higher proportion of progressive cataracts at higher dose levels. ${ }^{9}$ Long term studies are therefore needed to analyse the prevalence of lens changes following ionising radiation.

Haemangiomas are skin lesions of early childhood. Ionising radiation has been one of several treatment methods used in the past to facilitate regression. $\gamma$ Ray therapy for haemangiomas of the eyelids and skin according to a specified technique with surface application or implantation of radium needles ${ }^{10-12}$ were used between 1930 and 1964 at the department of radiotherapy at Sahlgrenska University Hospital, Gothenburg. During recent decades this therapy has been regarded as obsolete since it is known that cavernous and capillary haemangiomas in general undergo spontaneous regression. Radiation therapy of haemangiomas, therefore, has not been used in our hospital since 1964.

The purpose of our investigation was to make a cross sectional follow up study of adults who, as infants with haemangiomas, were treated with radium periocularly, and to evaluate the relation between cataract formation and levels of radiation in infancy in the long term. Thirty to 46 years after the radium treatment an examination of the eye was performed and, in addition, a semiquantitative assessment of lens opacities was carried out.

\section{Materials and methods}

SUBJECTS

From 1930 to 1964,76 children with haemangioma in the eyelid and surrounding tissues were treated with irradiation at the Radiological Clinic, Sahlgrenska University Hospital, 
Table 1 Patient and treatment characteristics together with lens opacity grading according to dose level

\begin{tabular}{|c|c|c|c|c|c|c|c|c|c|c|}
\hline \multirow[b]{3}{*}{$\begin{array}{l}\text { Case } \\
\text { No }\end{array}$} & \multirow{3}{*}{$\begin{array}{l}\text { Age at } \\
\text { treatment } \\
\text { (months) }\end{array}$} & \multirow{3}{*}{$\begin{array}{l}\text { Follow up } \\
\text { period } \\
\text { (years) }\end{array}$} & \multirow{3}{*}{$\begin{array}{l}\text { Treatment } \\
\text { time } \\
\text { (hours) }\end{array}$} & \multirow{3}{*}{$\begin{array}{l}\text { Average } \\
\text { distance } \\
(\mathrm{mm})\end{array}$} & \multicolumn{4}{|c|}{ Calculated dose (Gy) to the lens } & \multirow{2}{*}{\multicolumn{2}{|c|}{ Lens opacity grading }} \\
\hline & & & & & \multicolumn{3}{|c|}{ Treated side } & \multirow{2}{*}{$\begin{array}{l}\text { Untreated } \\
\text { side } \\
\text { Average }\end{array}$} & & \\
\hline & & & & & $\operatorname{Max}$ & Average & Min & & $\begin{array}{l}\text { Treated } \\
\text { side }\end{array}$ & $\begin{array}{l}\text { Untreated } \\
\text { side }\end{array}$ \\
\hline 9 & 10 & 35 & 2.3 & 12 & 1.00 & 1.05 & 1.15 & 0.03 & 1 & 0 \\
\hline 10 & 8 & 31 & 3.0 & 12 & 1.29 & 1.35 & 1.50 & 0.04 & 1 & 0 \\
\hline 11 & 2 & 32 & 2.3 & 10 & 1.12 & 1.40 & 1.64 & 0.03 & 2 & 0 \\
\hline 20 & 8 & 31 & 2 & 12 & 1.40 & 2.00 & 2.20 & 0.06 & 2 & 0 \\
\hline 1 & 3 & 37 & 1.5 & 6 & 2.93 & 2.19 & 1.72 & 0.02 & 2 & 0 \\
\hline 2 & 10 & 31 & 1.5 & 6 & 2.93 & 2.19 & 1.72 & 0.03 & 2 & 0 \\
\hline 3 & 13 & 43 & 1.8 & 6 & 3.41 & 2.56 & 2.01 & 0.04 & 2 & 0 \\
\hline 4 & 2 & 35 & 2.0 & 6 & 3.90 & 2.92 & 2.30 & 0.04 & 3 & 1 \\
\hline 16 & 2 & 33 & 1.7 & 8 & 3.59 & 2.97 & 2.33 & 0.09 & 3 & 1 \\
\hline 5 & 4 & 33 & 2.2 & 6 & 4.23 & 3.17 & 2.49 & 0.04 & 3 & 1 \\
\hline 17 & 2 & 34 & 1.8 & 8 & 3.93 & 3.26 & 2.56 & 0.06 & 3 & 1 \\
\hline 6 & 3 & 30 & 2.3 & 6 & 4.54 & 3.40 & 2.68 & 0.04 & 3 & 1 \\
\hline 18 & 9 & 43 & 2.2 & 8 & 4.64 & 3.84 & 3.02 & 0.12 & 3 & 1 \\
\hline 7 & 8 & 44 & 3.0 & 6 & 5.85 & 4.38 & 3.45 & 0.06 & 4 & 1 \\
\hline 8 & 10 & 46 & 3.0 & 6 & 5.85 & 4.38 & 3.45 & 0.06 & 4 & 1 \\
\hline 19 & 5 & 41 & 3.0 & 8 & 6.45 & 5.34 & 4.20 & 0.12 & 5 & 1 \\
\hline 14 & 8 & 43 & 2.0 & 6 & 7.38 & 5.62 & 4.50 & 0.08 & 4 & 1 \\
\hline 12 & 1.5 & 30 & 2.0 & 6 & 7.38 & 5.64 & 4.60 & 0.11 & 4 & 1 \\
\hline 13 & 2 & 44 & 2.4 & 6 & 8.92 & 6.80 & 5.56 & 0.07 & 4 & 1 \\
\hline 15 & 7 & 45 & 3.0 & 6 & 11.00 & 8.43 & 6.40 & 0.12 & 7 & 2 \\
\hline
\end{tabular}

during their first 13 months of life. Among these children 20 subjects were selected for follow up study according to the following criteria: a cavernous haemangioma of the eyelid localised at and within the orbital rim treated by application or implantation of radium-226 with one $(\mathrm{n}=11)$ or two needles $(\mathrm{n}=9)$; a patient record with detailed information concerning the treatment procedure and precise positioning of the source in relation to the eye. Cases treated with one or two needles were chosen in order to get the most reliable lens dose calculations and to decrease the uncertainty in dose estimates as described in an earlier study. ${ }^{13}$

From the national registries we traced the present addresses of these patients. They were invited by letter or telephone call to take part in this follow up examination; all patients ( $\mathrm{n}=$ 20) accepted. The age at the first treatment ranged from 2 to 13 months (median 6 months). They were $31-46$ years old when the follow up was made. The clinical data of the subjects are shown in Table 1.

\section{TREATMENT}

The application or implantation of radium-226 was made in the eyelid by using one or two needles. ${ }^{1011}$ Each needle contained $10 \mathrm{mg}$ radium-226 and was $14 \mathrm{~mm}$ long and $2.5 \mathrm{~mm}$ in diameter. The radium-226 source located in the centre of the needle was surrounded by two metal strata, one with $0.2 \mathrm{~mm}$ gold and one with $0.35 \mathrm{~mm}$ platinum. All radiation except the $\gamma$ radiation was filtered by the metal.

One or two needles were used depending on the size of the haemangioma. The implanted needle was placed, in general, parallel to the orbital rim on or within the haemangioma of the upper or lower eyelid. Detailed schematic drawings of the implantation geometry and detailed information about the treatment procedure were present for each patient in the hospital records. The treatment data with respect to treatment type, dose of irradiation, and localisation of the treatment area are listed in Table 1 according to increasing dose to the lens of the treated side.

In general, no shielding of adjacent tissues was used and the child was allowed to sit in the mother's lap or lie on a couch during treatment.

\section{CALCULATION OF DOSE OF $\gamma$ RADIATION TO THE} LENS

The lid haemangiomas were treated with implantation of one (cases 1-11) or two needles (cases 12-20) (Table 1). For haemangiomas located near the lid margin and the midline of the eyelid the distance from the most proximal portion of the anterior surface of the lens to the radiation source was calculated according to the following: anterior chamber depth and eyelid tissue was set equivalent to $5 \mathrm{~mm}$ (based on the Gullstrand's ${ }^{14}$ schematic eye at the age of 2-13 months), tissue oedema, and haemangioma tissue to $1 \mathrm{~mm}$ - that is, an average total distance of $6 \mathrm{~mm}$ from the source to the anterior lens surface.

In the other cases, where the haemangiomas were located closer to the orbital rim, an average distance according to anatomical location ranging from $8 \mathrm{~mm}$ to $12 \mathrm{~mm}$ was used for calculation. We calculated the average dose (Gy) of $\gamma$ radiation to the anterior surface of the lens of the treated eye from isodose curves based on the distance between the radiation source and the anterior surface of the lens as previously described. ${ }^{1015}$ The dose to the lens on the untreated side was similarly calculated. Interpupillary and intercanthal distances at different ages were extrapolated from the data reported by Pryor ${ }^{16}$ and Waitzman et al. ${ }^{17}$ For all cases a minimal and maximal dose was estimated (Table 1). The assumption was made that the actual distance could vary by plus or minus $1 \mathrm{~mm}$ during treatment as a result of local anaesthesia or eyelid movements. 
Table 2 Visual acuity and refraction of the irradiated compared with the fellow eye at follow up with increasing average dose to the lens of the treated side

\begin{tabular}{|c|c|c|c|c|c|}
\hline \multirow{2}{*}{$\begin{array}{l}\text { Case } \\
\text { No }\end{array}$} & \multirow{2}{*}{$\begin{array}{l}\text { Dose to the } \\
\text { lens }(G y)\end{array}$} & \multicolumn{2}{|c|}{ Visual acuity* } & \multicolumn{2}{|l|}{ Refraction } \\
\hline & & Treated side & Untreated side & Treated side & Untreated side \\
\hline 9 & 1.05 & 1.5 & 1.5 & $\pm 0-0.25100^{\circ}$ & \pm 0 \\
\hline 10 & 1.35 & 1.5 & 1.5 & -3.25 & -3.0 \\
\hline 11 & 1.40 & 1.05 & 2.00 & \pm 0 & \pm 0 \\
\hline 20 & 2.00 & 1.5 & 1.5 & $-4.75-0.75145^{\circ}$ & $-3.0-0.7555^{\circ}$ \\
\hline 1 & 2.19 & 1.0 & 1.5 & \pm 0 & \pm 0 \\
\hline 2 & 2.19 & 1.0 & 1.5 & \pm 0 & +0.5 \\
\hline 3 & 2.56 & 1.5 & 1.5 & -1.0 & -1.75 \\
\hline 4 & 2.92 & 1.5 & 1.5 & \pm 0 & \pm 0 \\
\hline 16 & 2.97 & 1.0 & 1.5 & -0.25 & -0.25 \\
\hline 5 & 3.17 & 1.5 & 1.5 & -1.5 & -0.75 \\
\hline 17 & 3.26 & 1.5 & 1.5 & \pm 0 & \pm 0 \\
\hline 6 & 3.40 & $0.2 \dagger$ & $0.2 \dagger$ & $-6.01 .540^{\circ}$ & $-7.01 .5180^{\circ}$ \\
\hline 18 & 3.84 & 1.0 & 1.0 & \pm 0 & \pm 0 \\
\hline 7 & 4.38 & 1.5 & 1.5 & $\pm 0-0.590^{\circ}$ & $\pm 0-0.5$ cyl $90^{\circ}$ \\
\hline 8 & 4.38 & 1.0 & 1.0 & -0.5 & \pm 0 \\
\hline 19 & 5.34 & 1.0 & 1.0 & \pm 0 & \pm 0 \\
\hline 14 & 5.62 & 1.0 & 1.0 & \pm 0 & \pm 0 \\
\hline 12 & 5.64 & 1.0 & 1.0 & $-0.5-1.0130^{\circ}$ & -0.25 \\
\hline 13 & 6.80 & 1.0 & 1.5 & \pm 0 & -0.5 \\
\hline 15 & 8.43 & 1.0 & 1.0 & $-0.75-0.7555^{\circ}$ & $\pm 0-1.75175^{\circ}$ \\
\hline
\end{tabular}

* Tested with best refraction at a distance of 5 metres with a LIC chart with approximate steps of $0.1 \log$ unit. †Bilateral, hereditary optic atrophy.

OPHTHALMIC EXAMINATION AT FOLLOW UP History taking followed a standardised protocol and questions were asked especially about potential cataractogenic factors-that is, diabetes, corticosteroid medication, $x$ ray to the head, etc. No special cataractogenic factor besides $\gamma$ radiation was documented in any of the cases besides bilateral myopia in cases 6 and 20 (Table 2).

Both eyes were examined by a trained ophthalmologist (GW) according to the standardised protocol. The observer was not masked as to the side of the radium needle(s). Testing of refraction and visual acuity (Table 2), followed by ophthalmoscopy of the fundus and slit-lamp microscopy of the lens and anterior segment of the eye were performed. A schematic drawing of the lens was made based on the microscope view in slit and retroillumination. Thereafter, photographs of the lens were taken with a Zeiss fundus camera and in slit and retroillumination. Colour film Ektachrome 200 was used. The patients were also photographed with the Oxford retroillumination cataract camera (Holofax Limited, UK) with a black and white Tri X Pan film.

Other changes of the eye and adnexa with no and minor subjective symptoms were also noted-that is, dry eye symptoms, telangiectasis of conjunctival vessels, loss of eyelashes, atrophy, and changed pigmentation of the skin of the eyelids (compare Donnenfeld et $a l^{2}$ ). In all cases these changes were only found on the treated side. In one case minor areas with defects of the underlying bone were noted on the treated side. No obvious relation to the calculated lens dose could be found and the changes were most marked in the area closest to the radiation source.

GRADING OF LENS OPACITIES

The presence of a localised opacity in the posterior subcapsular zone was documented by slit-lamp biomicroscopy and retroillumination photography. All lens opacities were graded by slit-lamp biomicroscopy using a modified
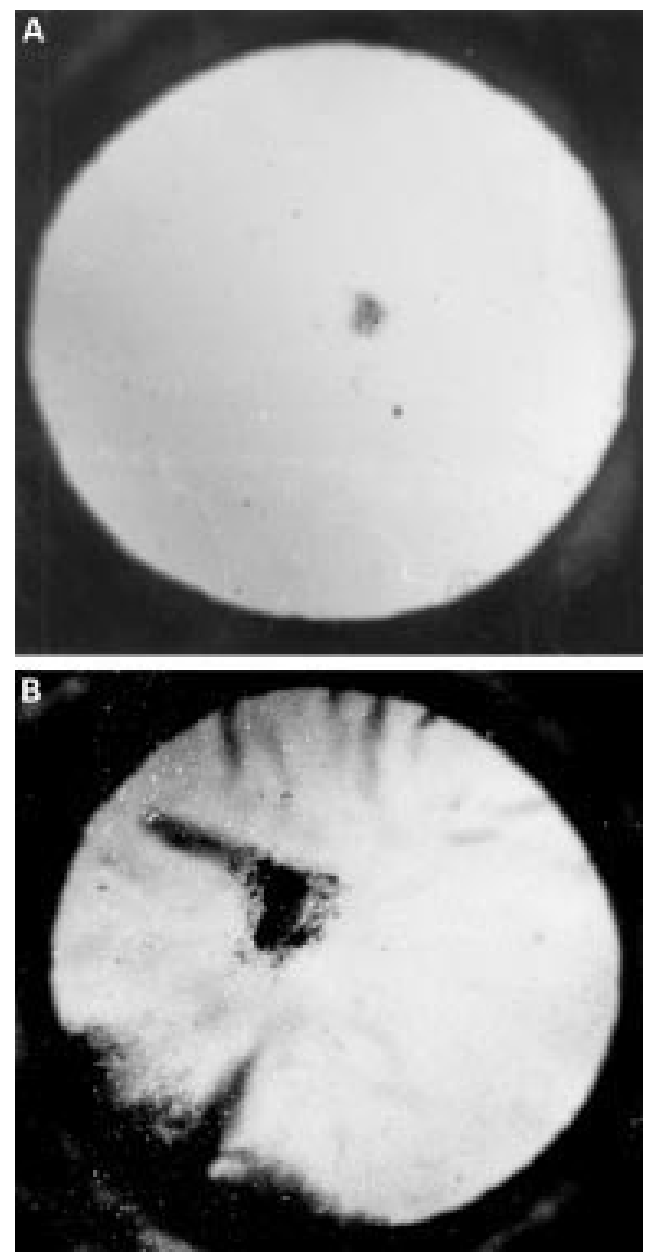

Figure 1 Retroillumination photography: (A) Grade 4 (case 8), (B) grade 7 (case 15) of radiation cataracts according to the description in the methods section.

version of classifications of radiation cataract in the posterior subcapsular region. ${ }^{5}{ }^{18}{ }^{19} \mathrm{By}$ combining classification criteria from these studies and, mainly, Tokunaga's study ${ }^{18}$ a hierarchical grading system for lens opacities of increasing severity was established primarily based on the increasing dimensions of the posterior subcapsular opacities and density together with other characteristics. The earliest change (grade 1) was punctate opacities and vacuoles grouped subcapsular in the posterior pole. They were clearly observable by slit-lamp biomicroscopy under high magnification but difficult to visualise by retroillumination photography. Grades 2-8 (slight to moderate radiation cataract), documented by retroillumination photography, were scored as follows: small, localised opacity in the posterior pole (grade 2); in combination with white punctate opacities and vacuoles in the posterior cortex (grade 3); wider plaque-like denser opacity (grade 4) (Fig 1(A)); denser and wider opacity (grade 5); dense, coarsely granular or tuff-like (tuff $=\mathrm{a}$ kind of volcanic, porous rock); opacity central to a wide, subcapsular plaque formation (grade 6); larger tuff-like opacity on a plaque-like formation (grade 7) with some anterior, cuneiform cortical opacities (Figs 1(B) and 2). Finally, grade 8 was a tuff-like opacity on a wider plaque formation occupying 


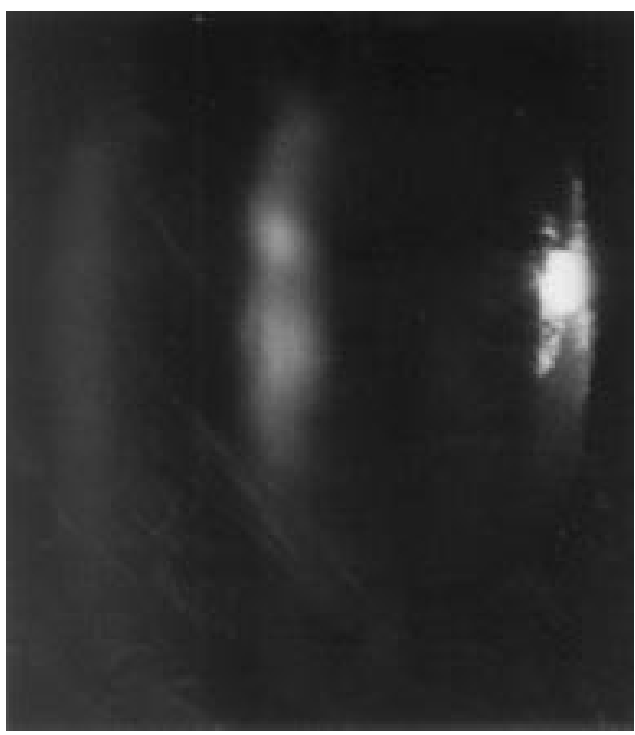

Figure 2 Slit-lamp photography of cataract grade 7 (case 15).

approximately one third of the area of the posterior subcapsular region within the dilated pupil. In addition, advanced cuneiform cortical opacities were observed at this stage. No case had severe radiation cataract changes (stages $9,10)$ characterised by advanced cataract. ${ }^{18}$

\section{Results}

The average dose of $\gamma$ radiation to the lens of the treated eye varied from approximately 1.1 to 8.4 Gy (Table 1). No other cataractogenic factor was documented in any of the cases studied. The patient (case 6, Table 2) with subnormal acuity had bilateral hereditary, dominant atrophy and no association with radiation is suspected.

All lenses of the eye on the treated side showed some changes in the posterior cortex adjacent to the posterior pole (Table 1). In two treated lenses (cases 9, 10, Table 1) receiving the lowest dose of $\gamma$ radiation (less than 1.4 Gy) only vacuoles and punctate opacities at slit-lamp observations were found in the posterior subcapsular zone. In the other 18 cases clear opacities could be documented photographically and the extent and density of the subcapsular opacities observed in retroillumination were related to the calculated dose (Fig 3). Most treated lenses (16/18) had slight radiation cataract (grades $2-4$ ) and only two in the highest dose range (more than $5 \mathrm{~Gy}$ ) had moderate (grades 5-8) radiation cataract (Table 1). No case of severe radiation cataract, according to Tokunaga's ${ }^{18}$ classification, was found in this study which included patients treated with only one or two radium-226 sources.

In the slit-lamp photographs it could be shown that the cataract extended from the subcapsular zone into the posterior cortex. In cataracts of higher grade two areas of increased light scatter separated by a clearer zone could be observed at least in the peripheral region of the cataract (Fig 2). In the central parts of the cataract closer to the visual axis the zones seem to aggregate. In cataracts of lower grades ( 4 or

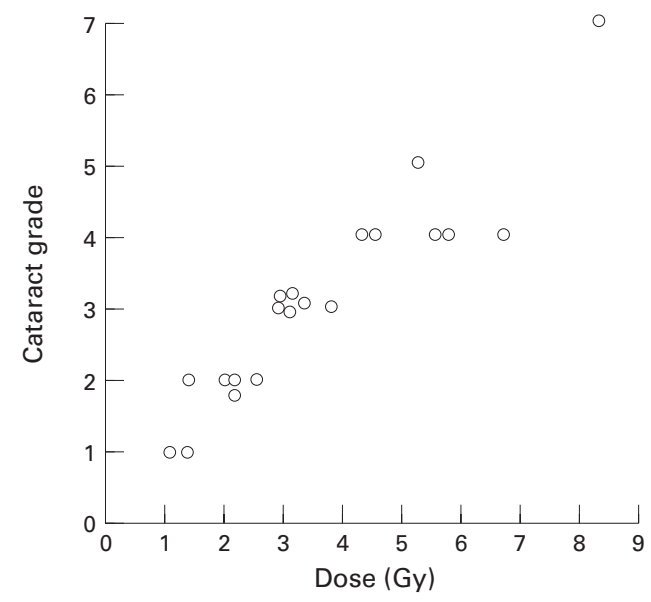

Figure 3 Cataract grade in the lens on the treated side as a function of dose.

less) the opacities were of more irregular location and were found in a zone extending from the subcapsular region into the deepest part of the posterior cortex.

The refraction and visual acuity on the treated side were within normal range and no clear differences from those of the untreated eyes were found. However, the case (no 15) with the most advanced cataract complained about glare.

The lenses of the eye on the untreated side received doses in the range 0.02-0.12 Gy (Table 1). Seven of the 20 cases showed no changes in the posterior pole of the lens on the untreated side (dose range 0.03-0.06). In the dose range $0.06-0.12$ Gy all but one of the lenses (10/11) had minor changes (grade 1 or 2 ) in the posterior pole. In all cases with cataract grade 3 or more on the treated side the lenses on the other side had small opacities.

\section{Discussion}

The main finding in this study of adults (31-46 years old), treated with radium-226 needles during infancy (2-13 months) as a result of haemangiomas within the orbital rim, is the high prevalence of posterior capsular opacities. If the earliest change consisting of punctuate opacities close to the posterior pole is included all had lens changes on the treated side. The cataract changes, according to a semiquantitative grading of the extent and density of the opacities, showed a relation between cataract grade and estimated dose to the lens in agreement with previous studies. ${ }^{6}{ }^{18}$

In agreement with studies of lens changes in adult patients receiving $\gamma$ radiation $^{6}$ our adult cases radiated in infancy show easily observable effects after doses of 2.0 Gy or more. The posterior subcapsular cataract formation was, in all cases, compatible with effects of radiation. ${ }^{518}$ However, since these changes cannot be considered pathognomonic of radiation cataract other causes must be ruled out. None of our cases had any other cataractogenic factors other than age between 31 and 46 years at examination and bilateral myopia in two cases. The possibility of presenile, unspecific cataract formation as a cause for the more 
subtle cataract grades (grades 1 and 2) is less probable since the contralateral lens in all cases, including the myopic case 20 receiving 2.6 Gy or less to the lens of the treated side showed no lens changes at the posterior pole. Although myopia is a recognised factor related to cataract, this association seems to be due mainly to the myopic shift found in early nuclear cataract rather than to a predisposition of the myopic eye to subcapsular cataract. ${ }^{20}$ Myopia as the cause of subcapsular cataract formation in cases 6 and 20 is therefore less probable. Furthermore, the extension of the subcapsular opacities into the posterior cortex found in this study is usually not seen in subcapsular cataracts acquired later in life.

The radiation cataract on the treated side was classified as slight to moderate according to Tokunaga's classification. ${ }^{18}$ No case diagnosed as severe radiation cataract was found in any of our cases with a maximal dose level around $8 \mathrm{~Gy}$. Children receiving total body irradiation of $10 \mathrm{~Gy}$ because of haematological malignancies before bone marrow transplantation all developed lens opacification and only $25 \%$ had an unaffected visual acuity after 3 years. ${ }^{21}$ Similarly, we have observed severe radiation cataract in cases that were treated with more than two needles and doses $10 \mathrm{~Gy}$ or more (Wilde, in preparation).

To determine the latency period before lens injury occurs is obviously of great importance. As a general rule, increasing the dose of radiation seem to be related to a shorter latency period before tissue effects are observed. ${ }^{22}$ As the lens grows throughout life at a steady rate with the youngest fibres most superficial there is a possibility of dating the onset of cataract by judging the depth of the cataract. ${ }^{23}$ In the present study changes could be observed in the deeper parts of the posterior, perinuclear cortex at least in the higher grades of cataracts indicating that damaged lens cells appeared probably within years after exposure to average lens doses of 5 Gy or more. This initial zone of radiation damage has, thereafter, been buried by growth of more normal fibres creating intervals with less light scatter. The presence of cataract in the subcapsular zone adjacent to the posterior capsule may be due to the persistence of damaged cells at the posterior pole. ${ }^{24}$ In other studies with long term follow up of radiation cataract several decades after radiation due to treatment with $x$ ray $^{24}$ or injected radium $-226^{25}$ it has also been observed that cataract formation is present in the posterior cortex covered by a zone of clear, more superficial cortex. These findings, in some cases of more clear zones superficial to the cataract in the posterior cortex, indicate that normal or less damaged lens fibres may be formed after an initial period.

The visual acuity and refraction seemed to be within normal limits. In agreement, Quist and Zachau-Christiansen ${ }^{7}$ found, in their follow up of cases treated 20-30 years earlier with radium therapy during childhood, that the radiation cataract as a rule does not essentially impair vision. In their investigation they point out that a relatively high number had an ametropia exceeding $+2 \mathrm{D}$. This was not found in our study. If some of the lenses in our study have been growth retarded as a result of radiation an emmetropising process may have compensated for eventual refractive changes.

To determine whether the cataract changes found in our study are stationary, a follow up study is needed. Merriam and Focht ${ }^{6}$ found that in a dose range of more than 6 Gy the majority showed progressive cataracts. Tokunaga ${ }^{18}$ reports that the radiation cataracts following the atomic bomb in Nagasaki did not progress except in cases with severe radiation cataract.

A follow up study of possible cataract progress even in a lower dose range would be of great interest since different possibilities exist. The original ionic radiation is known to give rise to DNA damage and a genotoxic effect, preferentially within the pre-equatorial, germinative zone of the lens epithelial cells. ${ }^{4}$ Some of these damaged cells may migrate to the equator and form damaged lens cells during the first years. Others may divide and form clones of damaged cells that may stay stationary for decades or eventually almost a lifetime in the germinative zone before starting migration and lens fibre differentiation. Damaged lens fibres may therefore be found at long intervals after the initial damage since no cells or debris are lost in the lens. Slow progress later in life can therefore occur as a result of the radiation damage in infancy. However, another scenario is also possible. The genotoxic, accumulating effects of an environmental nature on the lens epithelial cell-for example, ultraviolet light exposure or oxidative damage, may add to the previous radiation effect and produce accelerated age related cataract formation later in life.

The dose to the lens on the untreated side was calculated to be in the range $0.02-0.12$ Gy; $1 \%$ to $3 \%$ of that to the treated lens. Thirteen of 20 lenses $(65 \%)$ on the untreated side showed changes (exact $95 \%$ confidence limits 40.8 to $84.6 \%$ ). Slight changes consisting of vacuoles and subcapsular punctate opacities at the posterior pole were seen in the majority of lenses in the highest dose range 0.06-0.12 Gy. Even though age related, unspecific changes cannot be ruled out, the association with lens dose is to be noted in 10 out of 11 lenses with such changes in the highest dose range and no lens change in the low dose range. A cataract formation in the fellow eye as a result of a sympathetic granulomatous inflammatory reaction due to damage to the eye on the treated side is highly unlikely since no changes have been found in the posterior segment of those eyes. At low dose levels cultured bovine lens epithelial cells (the probable target for radiation) have been found to be sensitive to $x$ radiation with no evidence for thresholds at very low doses. ${ }^{26}$ It is therefore possible that a small percentage of lens epithelial cells in the germinative zone of the actively growing lens during infancy may be damaged due to a stochastic irradiation process. Survival studies of human normal epithelial cells and tumour cell lines of differing radiosensitivity are published after irradiation 
with single doses of $x$ rays. ${ }^{27}{ }^{28}$ At doses below 1.0 Gy the cells were more sensitive than predicted by back extrapolating the high dose response. A brief single exposure to $0.15 \mathrm{~Gy}$ has been reported to cause temporary sterility in men. ${ }^{29}$ It is therefore possible that the radiosensitivity of some proliferating lens epithelial cells in infancy is of a magnitude not very different from cells in adult spermatogenesis.

A careful calculation of the dose to the lens is important and the estimation of the distance between the lens and radiation source is critical. Even though the location of the radium-226 source within the orbital is well described in each patient record and our calculations included most variables that affect distance calculation, some degree of uncertainty is present. In a detailed phantom study imitating the treatment procedure with radium-226 needles used in our study, Lundell ${ }^{30}$ has made estimates of the absorbed dose in different organs in children with a mean age of 6 months treated for skin haemangiomas and discussed possible variables. The mean absorbed dose per 1000 mgh radium226 in the eye lens in infants treated for skin haemangioma in the head and neck was 2.99 cGy. ${ }^{30}$ The corresponding figure in our material from calculations done by Lundberg, ${ }^{15}$ is quite similar; 2.88 Gy per $1000 \mathrm{mgh}$ and equivalent to an average radiation source-lens distance of $5 \mathrm{~cm} .{ }^{15}$ The mean estimated dose to the lens in our group of infants with treatment at and within the orbital rim with $10 \mathrm{mg}$ of radium-226 (case 1-11) was 2.64 Gy. The mean treatment time was 2.26 hours and 1.16 Gy per $10 \mathrm{mgh}$, thus in a much higher dose range than the mean dose to the lens from treatments in the head and neck region in general, 0.291 Gy (data from Lundberg ${ }^{15}$ ).

In summary, the growing lens during infancy is highly radiosensitive with severity of lens damage in adult life related to dose of $\gamma$ radiation. The sensitivity was higher than expected on the untreated side. This is of great radiobiological interest and will be the object of further studies.

The authors would like to thank Professor Bengt Rosengren, Professor Magne Alpsten, and Drs Sture Lindberg, Bertil
Arvidsson, and Erik Holmberg for valuable discussions and Arvidsson, and Erik Holmberg for valuable discussions and
comments. This study was supported by grants from the Medicomments. This study was supported by grants from the Medical Research Counc
Blindas Vänner'.

1 Merriam Jr GR, Szechter A, Focht EF. The effects of ionizing radiations on the eye. Front Radiation Ther Oncol 1972;6:346-85

2 Rohrschneider W. Untersuchungen über die Morphologie und Entstehung der Röntgenstrahlenkatarakt beim Menschen. Arch $f$ Augenh 1932;106:221-54.
3 Cogan DG, Donaldsson DD, Reese AB. Clinical and pathological characteristics of radiation cataract. Arch Ophthallogical characteristics

mol 1952;47:55-70.
4 Worgul BV, Merriam Jr GR, Szechter A, Srinivasan BD. Lens epithelium and radiation cataract. Arch Ophthalmol 1976;94:996-9.

5 Cogan DG, Dreisler KK. Minimal amount of X-ray exposure causing lens opacities in the human eye. Arch Ophthalmol (Chicago) 1953;50:30-4.

6 Merriam Jr GR, Focht E. A clinical study of radiation cataract and the relationship to dose. Am 7 Roentgenol 1957;77: $759-85$.

7 Quist CF, Zachau-Christiansen B. Radiation cataract following fractioned radium therapy in childhood. Acta Radiol 1959;51:207-16.

8 Britten MJA, Halnan KE, Meredith WJ. Radiation cataract-new evidence on radiation dosage to the lens. $\mathrm{Br}$ fRadiol 1966;39:612-7.

9 Merriam Jr GR, Worgul BV. Experimental radiation cataract-its clinical relevance. Bull NY Acad Med 1983;59. $372-92$.

10 Strandqvist $M$. A new technique and dosage system for gamma ray therapy in surface application of radium. Acta Radiol 1939a;20:1-15.

11 Strandqvist M. Radium treatment of cutaneous cavernous haemangiomas, using surface application of radium tubes in glass capsules. Acta Radiol 1939b;20:185-209.

12 Lindberg S, Karlsson P, Arvidsson B, Holmberg E, Lundberg LM, Wallgren A. Acta Oncol 1995;34:735-40.

13 Notter G, Walstam R,Wikholm L. Radiation induced cataract after radium therapy in children. Acta Radiol 1966; 254:87-92.

14 Gullstrand A. Appendices to physiological optics. Vol 1. Helmholtz H, von, translated by Southall JPC. Rochester: Optical Society of America, 1924

15 Lundberg LM. Stråldoser till spädbarn som fått radiumbehandling för medfödda hemangiom. Report from Institute of Radiophysics. Göteborg: Sahlgrenska University Hospital, 1983.

16 Pryor HB. Objective measurement of interpupillary distance. Pediatrics 1969;44:973-7.

17 Waitzman AA, Posnick JCP, Armstrong DC, Pron GE. Craniofacial skeletal measurements based on computed tomography. Part II Normal values and growth trends. Cleft Palate-Craniofacial f 1992;29:118-28.

18 Tokunaga R. Atomic bomb radiation cataract in Nagasaki. Acta med nagasaki 1960;5:24-42.

19 Dodo T. Cataracts. F Radiat Res 1975; (Suppl) 132-7.

20 Brown NAP, Hill AR. Cataract: the relation between myopia and cataract morphology. $\mathrm{Br} \mathcal{F}$ Ophthalmol 1987;7:405-14.

21 Calissendorff $B$, Bolme P, el Azazi $M$. The development of cataract in children as a late side-effect of bone marrow transplantation. Bone Marrow Transplant 1991;7:427-9.

22 Donnenfeld ED, Ingraham HJ, Abramson DH. Effect of ionizing radiation on the conjunctiva, cornea, and lens. In: Sagerman RH, Alberti WE, eds. Radiotherapy of intraocular orbital tumors. Medical radiology series, 1993:261-70.

23 Brown N. Dating the onset of cataract. Trans Ophthal Soc UK 1976;96:18-23.

24 Hayes BP, Fischer RF. Influence of a prolonged period of low dosage $x$ rays on the optic and ultrastructural appearances of cataract of the human lens. B F Ophthalmol 1979;63:457-64.

25 Stefani FH, Mays ChW, Spiess H. Strahlenkatarakt nach Injektion von Radium-224. Fortschr Ophthalmol 1989;86: $32-7$.

26 Baumstark-Kahn C, Schneider J, Rink H. Radiation sensitivity of cultured bovine lens epithelial cells. Ophthalmic Res 1991;23:235-9.

27 Singh B, Arrand JE, Joiner MC. Hypersensitive response of normal human lung epithelial cells at low radiation doses. Int F Radiat Biol 1994;65:457-64

28 Lambin P, Fertil B. Malaise EP, Joiner MC. Multiphase survival curves for cells of human tumor cell lines: induced repair or hypersensitive subpopulation? Radiat Res 1994; 138:32-6.

29 IRCP Publication 60. Recommendations of the international commission on radiological protection. Oxford: Pergamon Press, 1990:15.

30 Lundell $M$. Estimates of absorbed dose in different organs in children treated with radium for skin hemangiomas. Radiat Res 1994;140:327-33. 